\title{
Le patrimoine en devenir des Tramways de l'Indre
}

\section{Bernard Moreau}

\section{OpenEdition}

\section{Journals}

Édition électronique

URL : https://journals.openedition.org/rhcf/2084

DOI : 10.4000/rhcf.2084

Éditeur

Rails \& histoire

Édition imprimée

Date de publication : 2 mai 2002

Pagination : 360-367

ISBN : 00996-9403

ISSN : 0996-9403

\section{Référence électronique}

Bernard Moreau, «Le patrimoine en devenir des Tramways de l'Indre », Revue d'histoire des chemins de fer [En ligne], 24-25 | 2002, mis en ligne le 21 avril 2015, consulté le 22 avril 2022. URL : http:// journals.openedition.org/rhcf/2084 ; DOI : https://doi.org/10.4000/rhcf.2084 


\section{Le patrimoine en devenir des Tramways de l'Indre}

Le réseau des Tramways de l'Indre, comme l'a dit M. du Pouget, comportait trois lignes. La première, ouverte en 1903, allait de Châteauroux à Valençay. La deuxième, complétée en 1904, reliait Issoudun à Vierzon, également dans le Cher. Enfin, Le Blanc-ArgentonChaillac est ouverte complètement en 1905'.

Le réseau comptait donc $180 \mathrm{~km}$ de voies métriques, une centaine d'arrêts, qu'il s'agisse de gares, de haltes, d'arrêts facultatifs..., et tous les équipements industriels nécessaires à l'exploitation.

Les uns comme les autres ont laissé des traces sensibles dans nos paysages d'aujourd'hui.

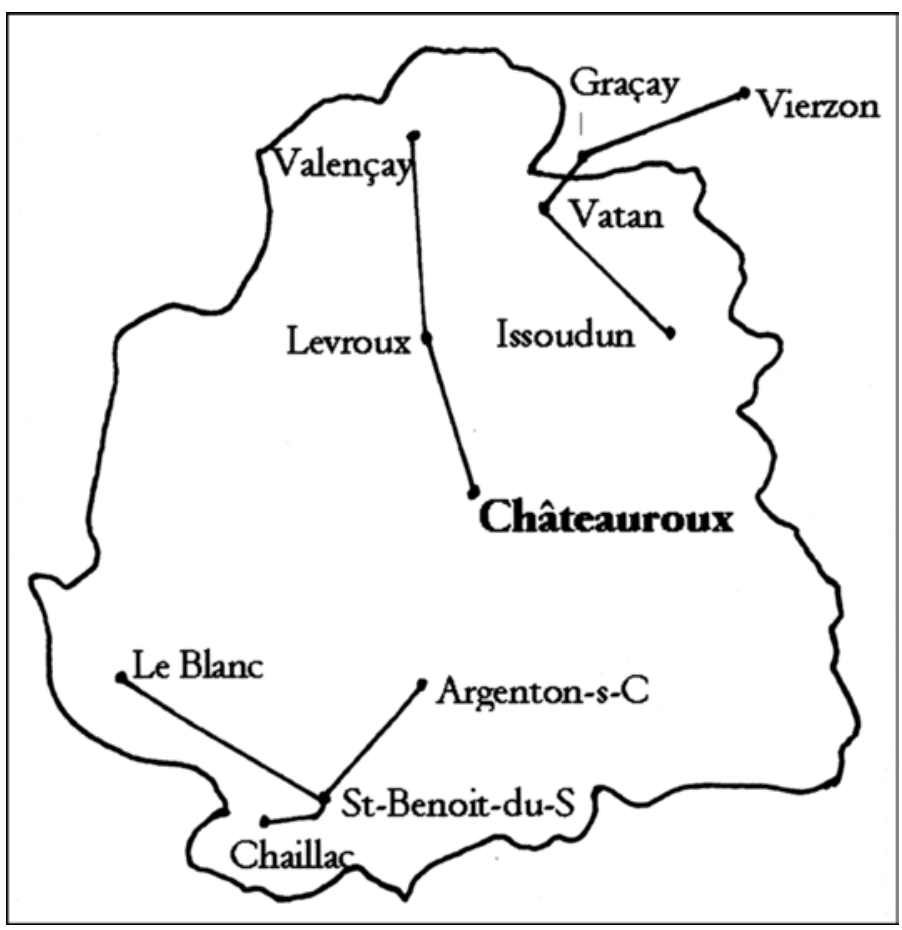

Figure 1 : Les lignes des Tramways de I'Indre.

${ }^{\circ}$ Bernard Moreau, 2000 
En novembre 1936, le conseil général de l'Indre vota la suppression du tramway à partir du $1^{\text {er }}$ avril 1937. En réalité, il sera prolongé jusqu'au 15 mai. Par la suite, quelques autorails et trains de marchandises circulèrent mais ne dépassèrent pas le début de l'été 1938.

A la fin du mois de juillet de cette année 1938, on vendit le matériel et les voies à la préfecture de l'Indre : trois locos, trois voitures, 22 wagons et 26 kilomètres de voies, déduction faite des sections de voie traversant les routes qu'on laissa en place pour éviter de trop importants travaux de voirie, soit 900 tonnes de ferraille. Les sommes récupérées devaient aider au financement des autobus, tracteurs et remorques qui devaient remplacer le tramway.

La section Argenton-Saint-Benoit-Chaillac fut conservée. Elle assurait le transport du minerai de fer extrait de Chéniers et Chaillac. On se rappela que, pendant la guerre 1914-1918, les envois de minerai pour les usines d'armement furent importants. Le conflit dont l'éventualité se précisait chaque jour davantage permettait de supposer qu'il en serait de même, mais la rapidité de l'invasion après la " drôle de guerre » déjoua les plans, le trafic fut peu important pendant les combats eux-mêmes et toute circulation cessa en septembre 1940. Pour éviter la vente « obligatoire » de la ferraille et du matériel aux occupants, le déclassement et la vente trainèrent volontairement en longueur, jusqu'en 1942. C'est ainsi que la dernière locomotive des trains de minerai partit pour l'Allemagne où elle circula avec une immatriculation allemande. Les Américains la récupérèrent et l'immatriculèrent à leur tour avant de la renvoyer à Châteauroux en 1946. Elle partit à la ferraille en 1948. Une autre loco, employée au mur de l'Atlantique, finit de pourrir, intransportable, au cimetière du matériel ferroviaire de Saint-Valérysur-Somme : c'est la 030 T Pinguely n ${ }^{\circ} 116$ de 1901 qui portait le $n^{\circ} 4$.

Après avoir vendu le matériel, on vendit les gares et les bâtiments d'exploitation. Dans un premier temps, les autobus et tracteurs furent abrités dans les dépôts de Déols, Vatan et Argenton. On les vendit ensuite lorsque les tracés et les conditions de circulation se modifièrent face à la concurrence des transporteurs privés.

\section{Soixante ans après la cessation d'activité du réseau, que reste-t-il des Tramways de l'Indre?}

Les installations de la gare du Blanc ont été remises à la SNCF. La direction de l'Équipement d'Argenton s'installa au dépôt des Baignettes et celle de Levroux à la gare. Les installations de SaintBenoit sont occupées par les services d'incendie. Le dépôt de Déols (fig. 2 et 3), racheté par un particulier, fut loué successivement à 
deux transporteurs locaux qui se modernisèrent par la suite. II est aujourd'hui inoccupé (fig. 4).

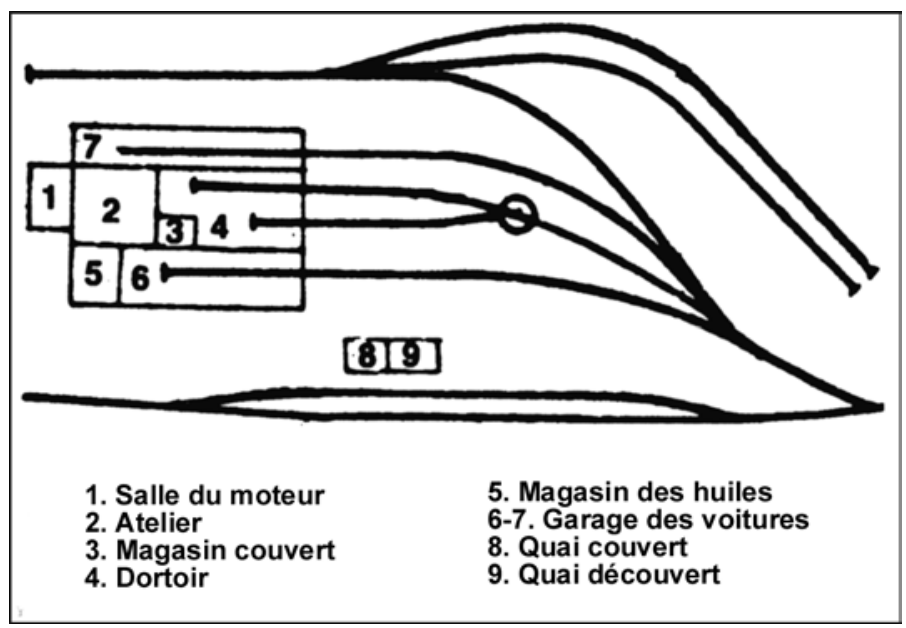

Figure 2 : Plan du dépôt de Déols.

๑) Bernard Moreau, 1989

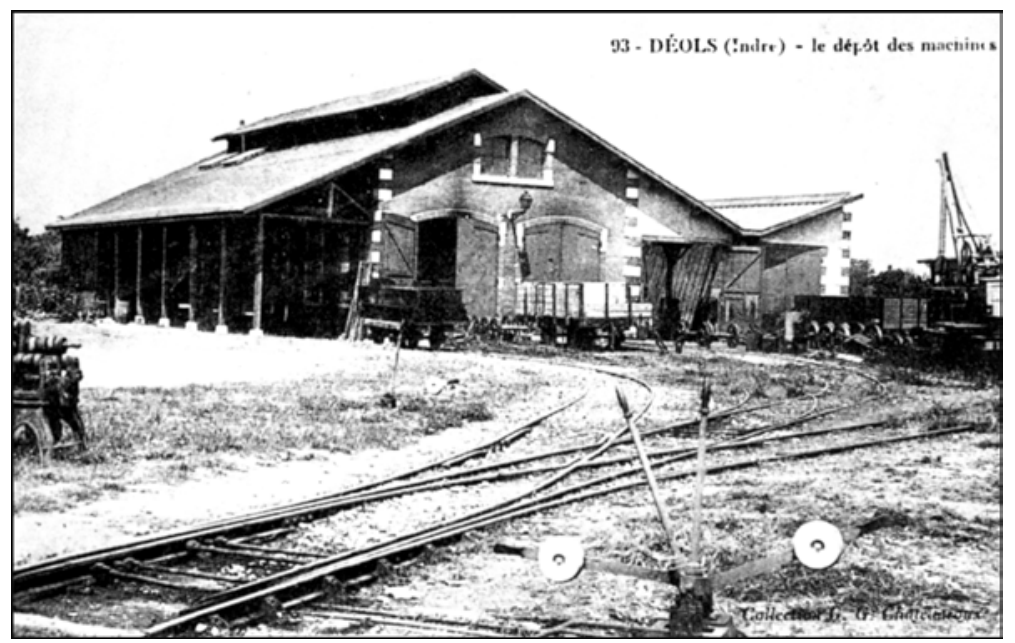

Figure 3 : Le dépôt de Déols en exploitation.

${ }^{\circ}$ G.G., Châteauroux 


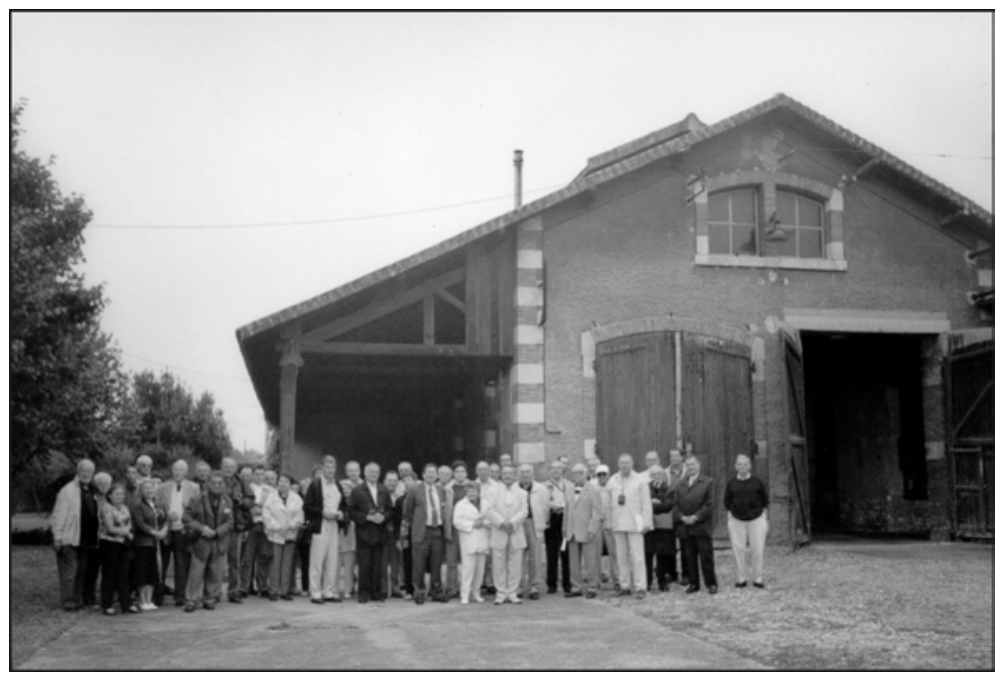

Figure 4 : Les participants au colloque réunis devant le dépôt de Déols, 8 septembre 2001.

(๑) AHICF / M.-N. Polino, 2001

Les gares ont subi différents « recyclages », devenant le plus souvent des résidences principales ou secondaires, artistement décorées ou conservant leur ancien caractère. Des abris ont été conservés comme arrêts pour les autobus, ils sont encore bien souvent debout, tel celui de la gare d'Argenton P.O., toujours en service. La gare de Châteauroux a été démolie en 1959, ainsi que les installations d'Issoudun, sauf la gare, qui survécut jusqu'en 1983. La gare de Vatan est intacte. Elle a été donnée à bail à la Société du Blanc-Argent jusqu'à 2003 et doit revenir ensuite à la ville de Vatan. L'ancienne remise abrite les cars qui font le service Vatan-Vierzon et la gare est occupée comme logement (fig. 5).

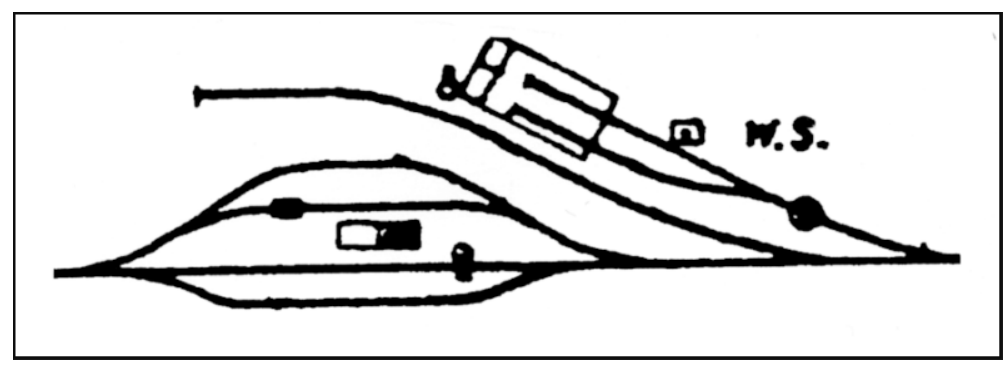

Figure 5 : Plan des installations de la gare de Vatan.

๑) Bernard Moreau, 1988 
Elle a la particularité d'offrir un ensemble cohérent et bien conservé puisqu'on peut y voir, outre le bâtiment voyageurs accolé à la halle marchandises en face de l'ancienne remise, la grue, un « édicule », un puits... (fig. 6).

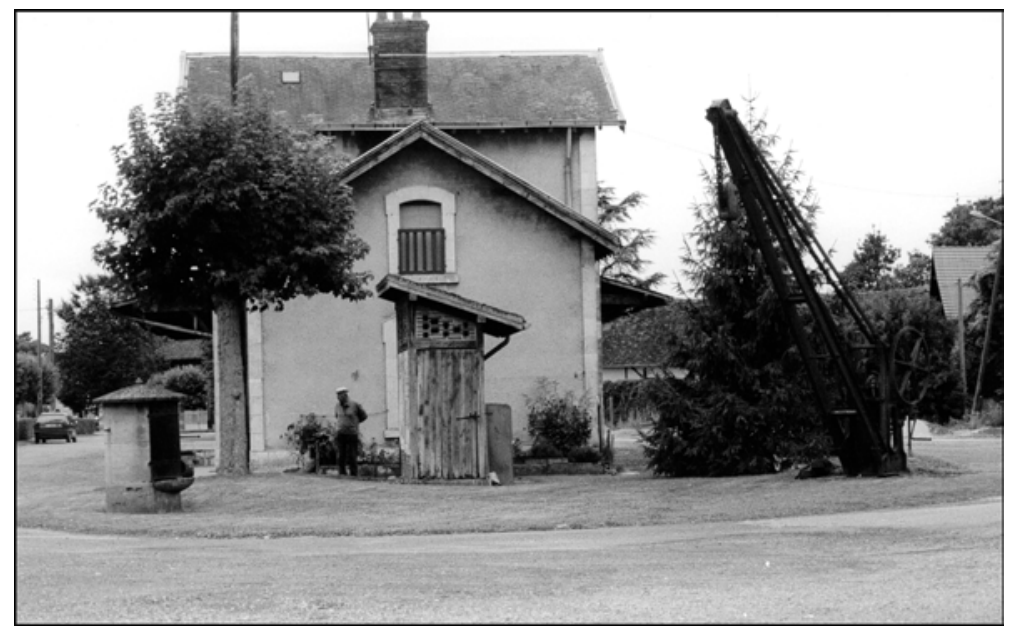

Figure 6: Les installations de la gare de Vatan, 8 septembre 2001.

( ) AHICF / M.-N. Polino, 2001

Cet ensemble est comparable en cela uniquement aux installations de la gare de Valençay, plus importantes puisqu'elles étaient destinées au Blanc-Argent et aux tramways (remise et dortoir, pont-bascule, réservoir d'eau, grue) mais, à la différence de celles-ci, il n'est pas protégé par une inscription à l'Inventaire supplémentaire des monuments historiques, ce que l'on peut regretter. D'autres gares ont été achetées par les municipalités et transformées en salles de réunion ou salles des fêtes. Il faut signaler spécialement la halte de Reboursin. Cette petite commune de 70 habitants n'avait plus d'église, sans doute depuis la Révolution. Le bâtiment a été transformé en chapelle en 1942 et dédié à Notre-Damede-la-Paix (fig. 7), avec une plaque commémorative pour les morts des deux guerres. 


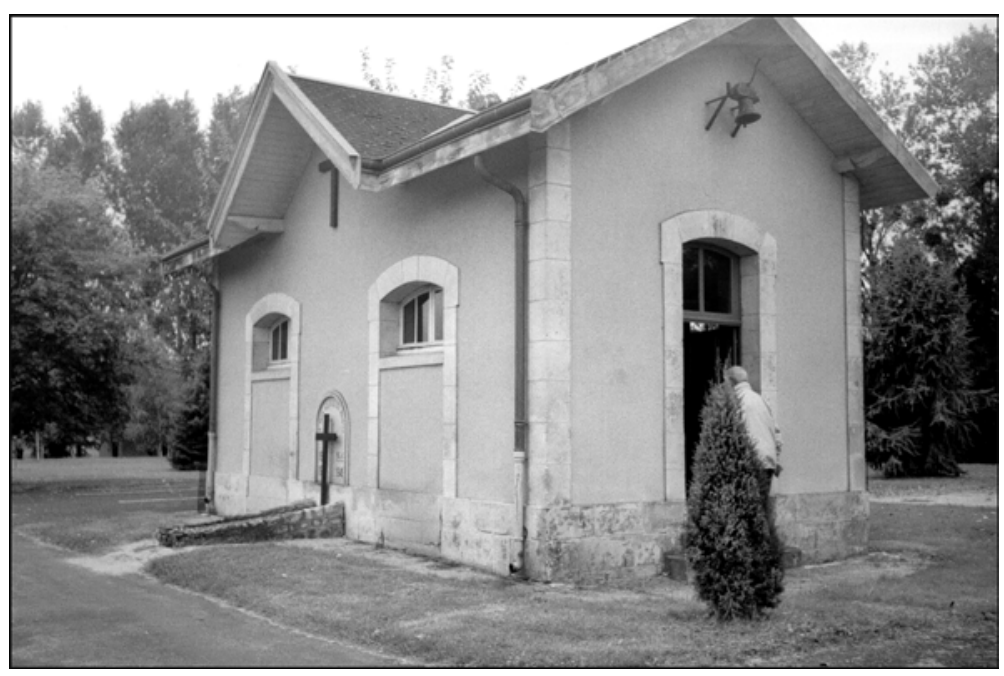

Figure 7 : La chapelle Notre-Dame-de-la-Paix, ancienne halte de Reboursin, 8 septembre 2001.

๑ AHICF / M.-N. Polino, 2001

À quelques kilomètres de là, l'importante station de Graçay (Cher) est devenue gendarmerie. Il n'y a guère que le bâtiment bordant la rue qui fait penser à une ancienne gare, tout le reste des installations a été supprimé. La gare de Moulins-sur-Céphons (sur la ligne ChâteaurouxValençay) est de même incluse, très bien repeinte, dans les bâtiments d'une entreprise. Au dépôt de Vierzon, le dortoir des locomotives trône au milieu des monuments en attente d'un marbrier funéraire.

Il subsiste çà et là des ouvrages d'art, ponts et ponceaux. Ils sont parfois en bordure de route, mais il faut aussi aller les chercher dans la nature en suivant le tracé des voies enlevées qui a été reconverti en chemins de promenade, souvent ombragés, tels celui de Saint-Benoitdu-Sault.

La gare de Valençay est la dernière étape de notre visite de la ligne des Tramways de l'Indre et la première de celle du Blanc-Argent.

Après un arrêt à la station de l'Hermitage (disparue) à l'entrée de Valençay, le tramway montait en ville où il avait un dernier arrêt facultatif au Champ-de-Foire, dont l'abri a été conservé, transformé en panneau d'affichage. En direction de Blois, il montait sur l'accotement gauche puis coupait la route et par une large courbe traversait en biais les voies du B.A. pour gagner son quai et les installations qui lui étaient réservées. Cette disposition fut l'objet de nombre de discussions et contrats et 
c'est pourquoi, lorsque la circulation des voyageurs fut ouverte jusqu'à Valençay le 12 avril 1903, le terminus fut pendant quelques mois « Tivoli », côté gauche, juste avant sa grande courbe. Il ne put pénétrer dans la gare qu’en fin d'année. Là aussi, la voie a été démontée et les hangars recyclés.

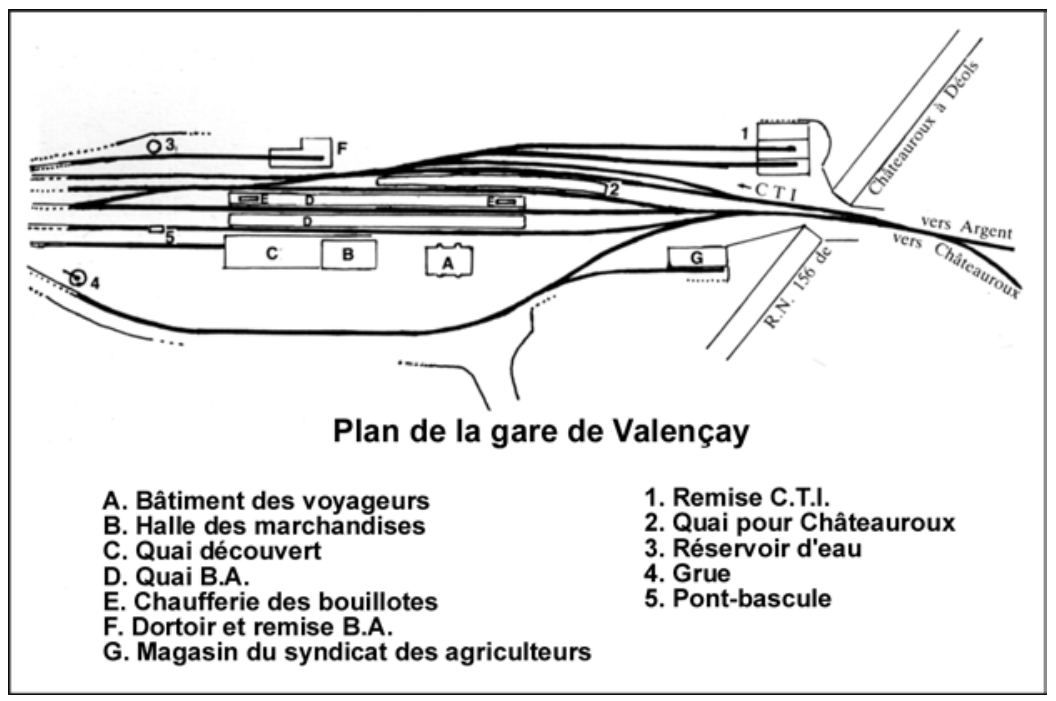

Figure 8 : Plan des installations de la gare de Valençay, où l'on voit la répartition des installations entre Le Blanc-Argent et la Compagnie des Tramways de I'Indre. ${ }^{\circledR}$ Bernard Moreau, 1989

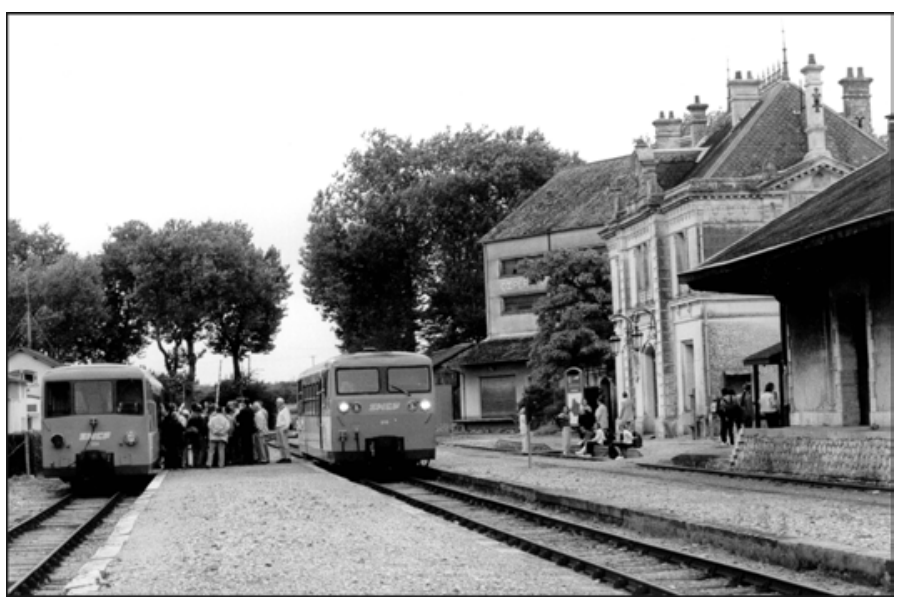

Figure 9 : La gare de Valençay (I.S.M.H. 18 janvier 1993). Le 8 septembre 2001 : départ de l'autorail régulier et autorail spécial mis à la disposition des partipants au colloque.

(๑) AHICF / M.-N. Polino, 2001 


\section{Note}

1- Voir, pour le détail de leur construction, Bernard Moreau, Le Tacot, petit train berrichon, tome 1, Histoire du tramway Issoudun-Vatan-Vierzon, Châteauroux, 1988, 124 p. ; tome 2, Tramways Châteauroux-Valençay et Le Blanc-Argenton-Chaillac, Issoudun, $1989,180 \mathrm{p}$. 
